\title{
Single-Tablet Regimens in HIV Therapy
}

\author{
Noemi Astuti · Franco Maggiolo
}

To view enhanced content go to www.infectiousdiseases-open.com

Received: January 16, 2014 / Published online: February 20, 2014

(C) The Author(s) 2014. This article is published with open access at Springerlink.com

\section{ABSTRACT}

Combined antiretroviral therapy (cART) has evolved considerably over the past decades leading to a better control of human immunodeficiency virus replication. Recently, regimens have evolved so as to simplify dosing frequency and reduce pill burden to improve adherence. Several national and international guidelines suggest antiretroviral (ARV) regimen simplification as a method of improving adherence. Decreased cART adherence has been associated with both patient-related factors and regimen-related factors. Adherence rates are statistically higher when simpler, oncedaily (OD) regimens are combined with smaller daily regimen pill burdens. The avoidance of selective non-adherence, where a patient takes part of a regimen but not the full regimen, is a further potential benefit offered by single-tablet regimens (STRs). Simplification of cART has

Electronic supplementary material The online version of this article (doi:10.1007/s40121-014-0024-z) contains supplementary material, which is available to authorized users.

N. Astuti · F. Maggiolo ( $\varangle)$

Unit of Antiviral Therapy, Division of Infectious

Diseases, AO Papa Giovanni XXIII, Bergamo, Italy

e-mail: franco31556@hotmail.com been associated with a better quality of life (QoL). Although tempered by other factors, better adherence, higher QoL and patients' preferences are all key points which might combine to assure long-lasting efficacy and durability of cART. All studies underlined the favorable tolerability profile of newer STRs. Three STRs are currently available. Tenofovir (TDF) plus emtricitabine (FTC)/efavirenz (EFV) was the first OD complete ARV regimen available as a STR. TDF plus FTC/rilpivirine is a second-generation STR. The most recently approved STR, TDF plus FTC/cobicistat/ elvitegravir, is the first non-nucleoside reverse transcriptase inhibitor-based STR. All of them have shown excellent efficacy; safety and tolerability have been improved by more recent formulations. Several other STRs are anticipated both combining completely different drugs, abacavir (ABC) plus lamivudine (3TC)/dolutegravir, utilizing innovative formulations of older drugs, tenofovir alafenamide fumarate, or taking advance of bioequivalent drugs, lamivudine (3TC) plus ABC/EFV. The future challenge would be to develop completely alternative STRs (including for example protease 
inhibitors or new molecules) to extend the advantages of simplicity to heavily pre-treated individuals.

Keywords: Adherence;

Combined antiretroviral therapy; Dolutegravir; Efavirenz; Elvitegravir; Human immunodeficiency virus; Infectious diseases; Single-tablet regimen; Rilpivirine; Quality of life

\section{INTRODUCTION}

Combination antiretroviral therapy (cART) has evolved considerably over the past two decades leading to better control of human immunodeficiency virus (HIV), preservation of the immune system and decreased incidence of opportunistic infections, malignancies and deaths. However, successful implementation of cART has been hampered by complicated regimens, high pill burden, drug-drug interactions and frequent short- and long-term adverse effects, leading to decreased adherence to prescribed regimens. Over time, the development of better-tolerated drugs with low or no dietary restrictions and fewer drug interactions has favored the success of cART and to further improve adherence, regimens have evolved so as to simplify dosing frequency and reduce pill burden. Early cART regimens were based on the administration of more than 25 pills, 3 times per day. Combination products consisted initially of partial regimens mostly combining two nucleoside reversed transcriptase inhibitors (NRTIs) such as zidovudine/lamivudine (3TC), abacavir (ABC)/ 3TC or tenofovir (TDF)/emtricitabine (FTC) or a boosted protease inhibitor (PI) lopinavir/ ritonavir (RTV), but, in 2006, the first singletablet regimen (STR), a combination of TDF/ FTC/efavirenz (EFV) became available [1] and, since then, STRs have been regarded as relevant tools to manage chronic HIV infection. The most advanced regimens used nowadays involve a single pill administered daily. The US guidelines now recommend that providers, when choosing between regimens of similar efficacy and tolerability, use once-daily (OD) regimens for treatment-naïve patients beginning cART, switch treatment-experienced patients receiving complex or poorly tolerated regimens to OD regimens, and use fixed-dose combinations (FDCs) and STRs to decrease pill burden [2].

The analysis in this article is based on previously conducted studies, and does not involve any new studies of human or animal subjects performed by any of the authors.

\section{STR and Adherence}

Several national and international guidelines underscore the importance of simplifying antiretroviral (ARV) regimens. The Spanish guidelines suggest that switching to a STR in stable patients currently receiving 2 NRTIs and a PI and RTV offers added advantages in terms of treatment adherence and that the use of STRs is the most efficient strategy to prevent selective treatment non-adherence [3], that is the possibility for a patient to consume less pills than those effectively prescribed. The Italian guidelines recommend the use of STRs and FDCs to improve durability of virologic suppression and to reduce the risk of developing resistance [4]. The European AIDS Clinical Society (EACS) guidelines recommend switching virologically suppressed patients for toxicity, to prevent long-term toxicity, and for simplification of a regimen.

Therapeutic switches must always be performed within a context of known viral resistance and it must always be kept in mind 
that any drug combination has its toxicological profile and that by switching it, it is possible to replace one set of toxicities with another. Nevertheless, it has been shown that the performance of patients who switched to an STR compared to patients remaining on a more complex regimen is superior, both in terms of virological response and persistence $[5,6]$.

Patient adherence is a problem in any chronic illness. A review of 76 studies across a wide range of therapeutic areas that measured adherence using electronic monitoring has revealed that compliance rates in clinical trials are lower than previously assumed and that the number of prescribed doses per day is inversely related to compliance. According to electronic monitoring methods, the overall adherence rate was $71 \pm 17 \%$. Adherence to OD regimens was significantly higher than with 3-times-daily and 4-times-daily regimens, which reinforces the principle of simplicity [7].

Decreased cART adherence is associated either with patient-related factors such as substance abuse, stress and depression, and with regimen-related factors. Regimen complexity includes the number of pills (pill burden), pill size, frequency and timing of doses, dietary and/or water requirements or restrictions, adverse events (AEs), medication storage requirements, number of prescriptions, number of copayments, refills, and medication bottles as well as the influence of these or other factors on the patient's lifestyle. Pill count, dosing frequency, and AEs have the greatest impact on patients' perceived ability to adhere to ARV medication regimens [8]. The exact rate of adherence necessary for cART treatment success is uncertain. Some studies indicate a minimum effective adherence rate of $80 \%$, although a higher level (at least 95\%) is considered ideal $[9,10]$. More recent experience has shown that the relationship between treatment adherence and viral load suppression as well as resistance development can vary among drug classes [11-13].

Several studies have shown that patients prefer OD regimens and simpler schedules [14-18]. A meta-analysis of 11 randomized, controlled trials on a total of 3029 subjects revealed that the adherence rate to cART was better with OD regimens, although the difference in adherence between OD and twice-daily (BID) regimens was modest (2.9\%) [19].

Nevertheless, adherence rates are statistically higher when simpler (OD) regimens are combined with smaller daily regimen pill burdens [20]. This statement is well elucidated by results of the ADONE (ADherence ONE pill; NCT \#00990600) study which just simplified treatment in HIV-controlled patients by reducing the number of pills without changing the pharmacological content. One month after the simplification from TDF + 3TC/FTC + EFV to TDF/FTC/EFV STR, the adherence rate increased significantly to $96.1 \%$ from a baseline value of $93.8 \% \quad(p<0.01)$; the increment was steadily maintained throughout the study (96.2\% at 6 months) [21].

It has been shown that patients on a fixeddose regimen of EFV/FTC/TDF were 2.1 times more likely to have complete adherence than patients on other regimens [22], that patients on a OD STR consistently achieve higher adherence levels than patients on $\geq 2$ pills per day regimens [23] and that STR was significantly better at achieving $\geq 90 \%$ adherence rates when compared with other multi-pill regimens (MPRs) ( $p<0.05$ all comparisons), despite an OD schedule or the use of FDCs [24].

With the methodological limits of a cohort study, in a commercially insured population of patients with HIV starting first-line cART, those beginning treatment with TDF/FTC/EFV had 
significantly better adherence and were more likely to be persistent with therapy than those beginning treatment with an EFV-based regimen other than TDF/FTC/EFV or a nevirapine (NVP)-based regimen [25]. Even among homeless or marginally housed patients, those receiving a single pill per day (TDF/FTC/EFV) had better virologic outcomes and a $26 \%$ increase in adherence, compared with those on MPRs [12].

The avoidance of selective non-adherence (taking some, but not all components in a given regimen) should be regarded as a further potential benefit of STRs. Selective nonadherence has been related to several clinical and economic drawbacks $[14,26,27]$. The COMPACT study [27] has shown that, independently from the type of cART, patients reported a complete non-adherence rate of about 20\%; however, patients on multi-drug regimens added an adjunctive $3-13 \%$ (according to the regimen) selective nonadherence. That made the difference statistically significant in favor of STRs. More relevant, patients receiving a STR had a more effective control of HIV replication (96\% of subjects below the limit of detection) and a better immune status (61\% above 500 cells/ mcl). Comparing partial (or selective) adherence of MPRs to STRs it has been demonstrated that complete non-adherence is relatively similar across regimens, while partial adherence is present with any MPR and doubles the risk of incomplete daily dosing [23].

Although the reduction of pill burden and dosing frequency has been associated to higher adherence rates, this may be only partially effective as drugs' tolerability and the occurrence of drug-related AEs may strongly undermine adherence. [16, 28-33].

Interestingly, a study in the Balearic Islands, showed a higher risk of AEs, some more severe in intensity as a consequence of the decision to administer ARV FDCs as separate components to reduce costs. Many of these AEs were neuropsychiatric disorders possibly related to EFV in stable patients who previously tolerated this drug. As a result, unlike the desired objective of cost saving, the disruption of ARV FDCs led to an increase of health care expenditure [34].

Adherence is also a cornerstone of persistence. Persistence is the length of time patients remain on a specific ARV regimen and is a key tenet to achieve long-term treatment success. NNRTI-based regimens exhibited greater persistence than PI-based ones. Among the specific regimens, TDF/FTC/EFV provided the longest persistence [35]. As successive ARV regimens have exhibited progressively shorter durability, optimizing the duration of the first regimen in treatment-naïve patients is of utmost importance. OD regimens had greater longevity than those taken BID or more frequently and the shift to newer, more convenient and better-tolerated therapeutic options has induced, over the last few years, a remarkable increase in the durability of first regimens [36].

\section{STR and Quality of Life (QoL)}

As cART options have increased and HIVinfected patients are living longer, the improvement or maintenance of health-related QoL has become an increasingly important goal of the management of chronic HIV infection. The maintenance of QoL passes through HIV medications use [37]. Simplification of cART has been shown to maintain or increase the QoL. Switching virologically suppressed subjects from their existing PI-based or NNRTI-based regimen to TDF/FTC/EFV STR was associated with maintained QoL and better treatment 
adherence. Patients referred an improved ease of use and an increment of treatment satisfaction after the switch to STR that was associated to a sustained improvement of several commonly encountered HIV-related symptoms [5].

One of the secondary objectives of the ADONE study was to verify the effect of the simplification strategy on QoL. The results confirmed an improvement of QoL over time from $68.8 \%$ to $72.7 \%(p=0.042)$ and this change was significantly associated with the perception of health status and presence, number and intensity of reported AEs $(p<0.0001)$. Also, QoL significantly influenced adherence $(p<0.0001)$ [21].

The switching boosted PI to rilpivirine (RPV) in combination with truvada as a STR (SPIRIT) study evaluated the switch from a PI-based cART to a STR (TDF/FTC/RPV) in chronically suppressed HIV patients. The study explored several patient-reported outcomes mostly dealing with symptoms often related to chronic therapies. After switching to STR, a general significant improvement of these symptoms was observed [6].

Better adherence, higher QoL and patients' preferences are all key points which may combine to assure long-lasting efficacy of cART.

\section{STR and Cost-Effectiveness}

Adherence is strictly correlated to hospitalization, as demonstrated in different studies. Completely adherent patients are less likely to be hospitalized or to require emergency room care than non-adherent patients [22].

Patients who achieve a 95\% adherence threshold have a significantly lower rate of hospitalization compared with patients who are non-adherent to therapy, regardless of their pill burden. Furthermore, patients who received a single pill per day were shown to be significantly less likely to be hospitalized than patients who received three or more pills per day [38].

In the COMPACT study [27], the type of therapy also influenced the total cost of illness. Patients treated with a STR showed association with the lowest cost. A selective non-adherence in a MPR of $3.5 \%$ increased the risk of hospitalization by 39\% thus further increasing management costs.

Patients on a STR have been associated with significantly lower monthly health care cost (US $\$ 605$ per patient per month $)(p<0.001)$ compared to patients on MPR. Differences were even greater (US \$922 per patient) $(p<0.001)$ when only treatment-naïve patients were examined. The use of OD STRs was associated with a $17 \%$ reduction in total health care costs, partly due to the significant reduction of hospitalization costs [23]. As already pointed out, cohort analyses have a few limitations and these results must be evaluated with caution as selection biases could play a role in the final outcome. As an example, patients with a lower CD4 nadir could have preferentially received a MPR and as the CD4 nadir is related to the risk of AEs and development of opportunistic pathologies their health care costs would naturally be higher. However, these concerns are somehow tempered by the consistency of results among different cohorts [23, 27].

The economic value of the switch from a two-pills-a-day TDF/FTC + EFV therapy to a STR (TDF/FTC/EFV) was evaluated by means of incremental cost-effectiveness ratio (ICER). The STR was the most cost-effective treatment strategy, with an ICER of $€ 22,017$ vs. $€ 26,558$ for the two-pills-a-day regimen [19]. Besides improving adherence and QoL as perceived by the patients, STRs allowed a $17 \%$ reduction of 
costs, corresponding to a $€ 4,541$ lower costeffectiveness ratio per quality-adjusted life-years (QALY) [39].

Similarly, the SPIRIT study showed that the use of the STR TDF/FTC/RPV was associated with an overall $16 \%$ cost reduction per subject through 24 weeks [6].

\section{Current STRs}

Three STRs are currently available. TDF/FTC/ EFV is a STR containing $300 \mathrm{mg}$ of TDF, $200 \mathrm{mg}$ of FTC and $600 \mathrm{mg}$ of EFV. It was the first OD complete ARV regimen available as a STR and is approved for use in all HIV patients, treatment naïve or experienced (alone as a complete regimen or in combination with other ARV agents for the treatment of HIV-1 infection in adults and pediatric patients 12 years of age and older) in the US, while only use in patients virologically suppressed more than 3 months is approved by the European Medicines Agency (EMA). TDF/FTC/RPV is a second-generation STR containing $300 \mathrm{mg}$ of TDF, $200 \mathrm{mg}$ of FTC and $25 \mathrm{mg}$ of RPV. It is licensed both in the US and in Europe for the use in HIV-infected subjects naïve or experienced (with a limitation referring to a viral load $<100,000$ copies/ml). More recently, TDF/FTC/ COBI (cobicistat)/EVG (elvitegravir) has been approved. It is the first non-NNRTI-based STR containing $300 \mathrm{mg}$ of TDF, $200 \mathrm{mg}$ of FTC, $150 \mathrm{mg}$ of EVG and $150 \mathrm{mg}$ of COBI. EVG is an integrase inhibitor that selectively inhibits the strand-transfer step of integration process of viral DNA into the nucleic acid of the host [40, 41]. COBI is a pharmacokinetic enhancer that does not exert any ARV activity [42].

TDF/FTC/EFV is currently one of the first choices for the treatment of HIV infection both in the US [43] and in the main European Guidelines [3, 44, 45]. It is the STR most widely used in clinical practice and the experience gained over years on the single components is much more extensive if compared to newer STR formulations. The US Guidelines have recently added TDF/FTC/COBI/ EVG as a preferred regimen and the European Guidelines have added TDF/FTC/RPV as a recommended regimen as well. Different studies have demonstrated that virologically suppressed patients receiving a wide array of NRTI backbones given with NNRTI- or PI-based therapies can be safely switched to the TDF/ FTC/EFV STR [16, 20, 21, 46].

Longer term data up to week 144 support the high durability of the use of TDF/FTC/EFV STR and a continued immunological recovery $[41,47]$.

TDF/FTC/EFV STR has been considered as the comparator arm in the trials leading to registration of new STRs. It showed high efficacy in naïve subjects coupled with a favorable toxicological profile (Tables 1, 2; [48-59]).

All components of the STRs were developed to be administered OD and possess long plasma and intracellular half-lives that are congruent one to the other which may provide an additional pharmacologic advantage in the case of occasionally missed doses as the unintentional functional monotherapy is prevented and the regimen genetic barrier is enhanced.

Two cohort studies $[60,61]$ have considered this aspect drawing similar conclusion. They studied the change in the prevalence of mutations for any component of the TDF/FTC/ EFV STR after the introduction in the market of the STR itself compared to the prevalence of the same viral mutations in the period these drugs were used as single components. Although both studies may suffer methodological drawbacks and selection bias impossible to rule out, they 
Table 1 Tolerability profile of single-tablet regimens (STRs)

\begin{tabular}{|c|c|c|c|c|c|}
\hline $\begin{array}{l}\text { Reason for } \\
\text { drug } \\
\text { discontinuation }\end{array}$ & $\begin{array}{l}\text { TDF/FTC/EFV } \\
\text { STaR (\%) } \\
(\mathbf{n}=392)\end{array}$ & $\begin{array}{l}\text { TDF/FTC/ } \\
\text { EFV 102 (\%) } \\
(\mathbf{n}=352)\end{array}$ & $\begin{array}{l}\text { TDF/FTC/RPV } \\
\text { STaR (\%) } \\
(\mathbf{n}=394)\end{array}$ & $\begin{array}{l}\text { TDF/FTC/COBI/ } \\
\text { EVG } 102(\%) \\
(n=348)\end{array}$ & $\begin{array}{l}\text { TDF/FTC/COBI/ } \\
\text { EVG } 103(\%) \\
(n=353)\end{array}$ \\
\hline Renal events & 0 & 0 & 0 & 2.0 & 0.8 \\
\hline $\begin{array}{l}\text { Rash and skin } \\
\text { reactions }\end{array}$ & 0.5 & 1.4 & 0 & 0 & 0 \\
\hline Diarrhea & 0.5 & 0 & 0 & 0 & 0.6 \\
\hline Nausea & 0 & 0 & 0 & 0 & 0.3 \\
\hline Vomiting & 0 & 0 & 0 & 0 & 0.3 \\
\hline Fatigue & 0.5 & 0.6 & 0 & 0.3 & 0 \\
\hline Pyrexia & 0.5 & 0 & 0 & 0 & 0.6 \\
\hline Hepatitis C & 0 & 0 & 0 & 0 & 0.3 \\
\hline Dizziness & 1.5 & 0 & 0 & 0 & 0 \\
\hline $\begin{array}{l}\text { Abnormal } \\
\text { dreams }\end{array}$ & 1.8 & 0.6 & 0 & 0 & 0 \\
\hline Insomnia & 1.0 & 0.6 & 0.3 & 0 & 0 \\
\hline Depression & 2.0 & 1.1 & 0 & 0.3 & 0 \\
\hline Suicidal ideation & 0.8 & 0 & 0 & 0 & 0 \\
\hline
\end{tabular}

Reasons for drug discontinuation due to intolerance (\%) as reported by the studies STaR, 102 and 103.96 week results $C O B I$ cobicistat, $E F V$ efavirenz, $E V G$ elvitegravir, $F T C$ emtricitabine, $R P V$ rilpivirine, $S T a R$ single-tablet RPV, TDF tenofovir

both concluded that there was a temporal association between the incremental use of the STR and the decreased prevalence of signature mutations. The French study conducted between 2005 and 2010 showed that the overall prevalence of resistance associated mutations to TDF, 3TC/FTC and EFV decreased over time, in the same period the use of TDF almost doubled without any increment of the K65R mutation; the use of 3TC was more than halved while the use of FTC increased from $8 \%$ to $53 \%$ with a decrease in M184 V/I prevalence; the introduction and the expansion of the use of EFV as a STR was associated with a decrease of the prevalence of the $\mathrm{K} 103 \mathrm{~N}$ [60]. These decreases may show the importance of utilizing FTC instead of 3TC in combination with TDF, as well as to the importance of the STR combination.

The virological efficacy of RPV has been demonstrated in naïve patients in different studies $[48,49]$ (Table 2). The pooled data of the early capture HIV cohort study (ECHO) and [treatment of HIV RPV vs. EFV trials (THRIVE)] indicated RPV as non-inferior to EFV both at 48 and 96 weeks. A slightly higher incidence of virologic failures was observed with RPV (14\%) vs. EFV (8\%), this difference mostly accumulated in the first 48 weeks of therapy, while failures were comparable afterwards, and occurred primarily in those with VL $>100,000$ $\mathrm{c} / \mathrm{mL}$. The virologic failure difference reduced in 
Table 2 Efficacy profile of different single-tablet regimens (STRs) or regimens potentially leading to a STR in naïve patients

\begin{tabular}{|c|c|c|c|c|c|}
\hline Reference & STR regimen & $\begin{array}{l}\text { Success } \\
\text { rate }(\%)\end{array}$ & Control arm & $\begin{array}{l}\text { Success } \\
\text { rate }(\%)\end{array}$ & $\begin{array}{l}\text { Follow-up } \\
\text { (weeks) }\end{array}$ \\
\hline $\begin{array}{l}\text { Cohen et al. (ECHO, THRIVE) } \\
{[48]}\end{array}$ & 2 NRTIs + RPV & 84 & 2NRTIs + EFV & 82 & 48 \\
\hline Cohen et al. (STaR) [49] & $\mathrm{TDF} / \mathrm{FTC} / \mathrm{RPV}$ & 86 & TDF/FTC/EFV & 82 & 48 \\
\hline Cohen et al. [50] & TDF/FTC/RPV & 78 & TDF/FTC/EFV & 78 & 96 \\
\hline Cohen et al. [41] & $\begin{array}{l}\text { TDF/FTC/COBI/ } \\
\text { EVG }\end{array}$ & 90 & TDF/FTC/EFV & 83 & 48 \\
\hline Sax et al. [51] & $\begin{array}{l}\text { TDF/FTC/COBI/ } \\
\text { EVG }\end{array}$ & 88 & TDF/FTC/EFV & 84 & 48 \\
\hline Zolopa et al. [52] & $\begin{array}{l}\text { TDF/FTC/COBI/ } \\
\text { EVG }\end{array}$ & 84 & TDF/FTC/EFV & 82 & 96 \\
\hline Wohl et al. [53] & $\begin{array}{l}\text { TDF/FTC/COBI/ } \\
\text { EVG }\end{array}$ & 80 & TDF/FTC/EFV & 75 & 144 \\
\hline De Jesus et al. [54] & $\begin{array}{l}\text { TDF/FTC/COBI/ } \\
\text { EVG }\end{array}$ & 90 & $\begin{array}{l}\mathrm{TDF}+\mathrm{FTC}+\mathrm{ATV} / \\
\mathrm{rtv}\end{array}$ & 87 & 48 \\
\hline Rockstroh et al. [55] & $\begin{array}{l}\text { TDF/FTC/COBI/ } \\
\text { EVG }\end{array}$ & 83 & $\begin{array}{l}\mathrm{TDF}+\mathrm{FTC}+\mathrm{ATV} / \\
\mathrm{rtv}\end{array}$ & 82 & 96 \\
\hline Clumeck et al. [56] & $\begin{array}{l}\text { TDF/FTC/COBI/ } \\
\text { EVG }\end{array}$ & 78 & $\begin{array}{l}\mathrm{TDF}+\mathrm{FTC}+\mathrm{ATV} / \\
\mathrm{rtv}\end{array}$ & 75 & 144 \\
\hline Raffi et al. (SPRING 2) [57] & 2NRTIs + DTG & 81 & 2 NRTIs + RAL & 76 & 48 \\
\hline Feinberg et al. (FLAMINGO) [58] & 2NRTIs + DTG & 90 & $2 \mathrm{NRTI}+\mathrm{DRV} / \mathrm{rtv}$ & 83 & 48 \\
\hline Walmsley et al. (SINGLE) [59] & $3 \mathrm{TC} / \mathrm{ABC}+\mathrm{DTG}$ & 88 & TDF/FTC/TDF & 81 & 48 \\
\hline
\end{tabular}

Success rate is virologic success evaluated according to the US Food and Drug Administration snapshot analysis definition $A B C$ abacavir, $A T V$ atazanavir, $C O B I$ cobicistat, $D R V$ darunavir, $D T G$ dolutegravir, $E F V$ efavirenz, $E V G$ elvitegravir, $F T C$ emtricitabine, $N R T I$ nucleoside reversed transcriptase inhibitors, $R A L$ raltegravir, $R P V$ rilpivirine, $r t v$ ritonavir, $S T R$ singletablet regimens, $T D F$ tenofovir, $3 T C$ lamivudine

the open-label single-tablet RPV (STaR) study that used the STR formulation, suggesting the relevance of the STR on adherence [49]. In the registrative studies, the subgroups of patients with baseline HIV-RNA $>100,000$ copies $/ \mathrm{mL}$ showed higher rates of virological failures and more frequent emergence of NNRTI and NRTI resistance including the $\mathrm{E} 138 \mathrm{~K}$ resistance mutation that causes cross-resistance with etravirine (ETR) [50]. These studies have justified the approved indication limiting the use of TDF/FTC/RPV STR to patients with lower baseline viremia. In the open-label STaR study, the TDF/FTC/RPV STR favorably compared with the TDF/FTC/EFV STR. Considering the totality of patients the second-generation STR was noninferior to the control arm and a post hoc analysis stratified according to the baseline viral 
load, revealed that TDF/FTC/RPV was superior to $\mathrm{TDF} / \mathrm{FTC} / \mathrm{EFV}$ in patients with viral load $<100,000$ copies/mL [49].

All studies underlined the favorable tolerability profile of TDF/FTC/RPV (see Table 1) [48, 49]. RPV was well tolerated, demonstrating fewer drug discontinuations, and reduction in central nervous system (CNS) and rash AEs, when compared to EFV.

These characteristics were further explored in a few small switch studies. In a cohort of patients chronically and successfully treated with TDF/FTC/EFV STR, the switch to TDF/ FTC/RPV STR obtained a significant and steady reduction of CNS-related symptoms such as dizziness $(p=0.008)$, depression $(p=0.029)$, insomnia $(p=0.001)$, anxiety $(p=0.021)$, confusion $(p=0.005)$, impaired concentration $(p=0.008)$, somnolence $(p=0.003)$, aggressive mood $(p=0.034)$ and abnormal dreams $(p<0.001)$ that turned out in a significant improvement in the quality of sleep $(p<0.001) \quad$ [62]. A similar experience conducted in the US concluded that switching from TDF/FTC/EFV to TDF/FTC/RPV appears to be a safe and efficacious option in virologically suppressed HIV-1-infected subjects who experience EFV intolerance and wish to remain on a STR [63].

In a larger controlled study in experienced patients, switching to TDF/FTC/RPV was noninferior to remaining on a PI/RTV + 2NRTIs regimen with a lower rate of virological failure in the TDF/FTC/RPV arm. Mutations developed in $<1 \%$ of subjects switched to TDF/FTC/RPV and after the switch an improvement in fasting lipids, including total cholesterol, low-density lipoproteins (LDL) cholesterol, triglycerides and total cholesterol/high-density lipoproteins (HDL) ratio was observed at week 24 and week 48 [6].
TDF/FTC/COBI/EVG is the most recent available STR, recommended as preferred in the Department of Health and Human services (DHHS) Guidelines for naïve HIV-infected patients with creatinine clearance $(\mathrm{CrCl})$ $>70 \mathrm{~mL} / \mathrm{min}[43,45,64]$.

The integrase inhibitor EVG can be administered OD. The speed of viral suppression observed with TDF/FTC/COBI/ EVG is consistent with the potency of HIV integrase inhibitors and robust COBI-boosted EVG exposures $[41,65]$. TDF/FTC/COBI/EVG has shown to be non-inferior for safety and efficacy to TDF/FTC/EFV at 48 [51], 96 [52] and 144 weeks [53] in a controlled, randomized trial enrolling 700 HIV-positive cART-naïve subjects (Table 2). At week $48,87.6 \%$ of the patients receiving TDF/FTC/COBI/EVG had HIV-RNA concentrations $<50$ copies $/ \mathrm{mL}$ vs. $84.1 \%$ of those receiving TDF/FTC/EFV [57]. HIV-RNA concentrations $\quad<50$ copies $/ \mathrm{mL}$ were maintained at week 144 in $80 \%$ of the TDF/ FTC/COBI/EVG arm vs. $75 \%$ in the TDF/FTC/ EFV arm, testifying for durability [53]. Very few patients in the TDF/FTC/COBI/EVG arm discontinued because of AEs, $4 \%$ at week 48 [51] and 5\% at week 96 and 6\% at week 144 $[52,53]$. The most common AEs observed in the TDF/FTC/COBI/EVG arm were nausea and an increase of serum creatinine concentration with a decrease in estimated glomerular filtration rate (eGFR). COBI is associated with reduced active secretion of creatinine in the renal tubules leading to initial rises in creatinine levels in the first 2-4 weeks [52]. Because of this, only patients with a $\mathrm{CrCl}>70 \mathrm{~mL} / \mathrm{min}$ were included in the registrative studies and consequently the use of COBI is currently allowed only in patients with $\mathrm{CrCl}>70 \mathrm{~mL} /$ min. Large pharmacovigilance programs on this enhancer should be considered to look at its 
long-term impact on renal function, not limiting data to just eGFR changes.

A second, large (715 enrolled patients), non-inferiority double-blind trial compared TDF/FTC/COBI/EVG to atazanavir (ATV)/ $\mathrm{RTV}+\mathrm{FTC/TDF}$. The primary endpoint was the proportion of patients suppressed at week 48 [54], but secondary endpoint week 96 [55] and 144 [62] data are available. At week 48, $89.5 \%$ of the patients receiving TDF/FTC/COBI/ EVG had HIV-RNA concentrations < 50 copies/ $\mathrm{mL}$ vs. $86.8 \%$ of those receiving ATV/ RTV + FTC/TDF [60]. At week 144, the figures were $78 \%$ and $75 \%$ [56]. As for the previous study, the rate of discontinuation in the TDF/ FTC/COBI/EVG arm due to AEs was very low (3.7\% at week 48) [54] (Table 1). Furthermore, the TDF/FTC/COBI/EVG-treated patients had statistically lower increases in fasting triglycerides, and a lower percentage of subjects experienced alanine aminotransferase (ALT), aspartate aminotransferase (AST) or bilirubin elevations when compared with ATV/RTV + TDF/FTC-treated patients.

As for resistances, in the 102 study [51], 2\% of patients in the TDF/FTC/COBI/EVG arm failed with resistance inducing mutations, usually to both NRTIs and EVG. The result was comparable to that observed in the TDF/FTC/ EFV arm. The integrase gene resistance mutations observed were E92Q, Q148R and $\mathrm{N} 155 \mathrm{H}$, which confer decreased susceptibility both to EVG and raltegravir and a potential lowlevel resistance to dolutegravir, and T66I, which preserves both raltegravir and dolutegravir (DTG).

\section{Future Perspectives}

An interesting STR that is anticipated is the combination ABC/3TC/DTG.
Dolutegravir is an unboosted integrase inhibitor that has been effectively and safely used for the treatment of HIV-infected naïve (with 2 NRTIs) and experienced patients (with optimized background regimen) [57-59, 66, 67], (Table 2). DTG has shown to be effective with $\mathrm{ABC} / 3 \mathrm{TC}$ or TDF/FTC regardless of blood level HIV-1 RNA [66], although the number of patients on $\mathrm{ABC} / 3 \mathrm{TC}$ with high viral load is limited. The efficacy of DTG has been compared to raltegravir in the SPRING-2 (NCT\#01227824) study; both associated with 2 NRTIs in cARTnaïve patients: DTG $50 \mathrm{mg}$ OD was as effective as raltegravir $400 \mathrm{mg}$ BID at 96 weeks (81\% vs. $76 \%)$. In the NRTI backbone comparison at 96 weeks those on DTG with ABC/3TC had efficacy rates of $74 \%$ compared to those on TDF/ FTC of $86 \%$. [57]. DTG has also been compared to a boosted-PI, both associated with 2 NRTIs (TDF/FTC or ABC/3TC). The open-label FLAMINGO (NCT\#01449929) study has shown the superiority in efficacy of DTG compared to darunavir (DRV)/RTV at week 48, driven by higher discontinuations in the DRV arm. Virologic failure was observed in 2 patients (1\%) on each arm without treatment-emergent resistance in either arm. The most common AEs were diarrhea with DRV/RTV and headache with DTG, while treatment-related study discontinuations were low (1\% on DTG arm, $4 \%$ on DRV/RTV arm) [58].

In the SINGLE (NCT\#01263015) study, enrolling naïve patients, DTG $50 \mathrm{mg}+\mathrm{ABC} /$ 3TC had a better safety profile and was more effective through 48 weeks than TDF/FTC/EFV. The time to reach HIV-RNA $<50$ copies/mL was 28 days with DTG vs. 84 days with EFV $(p<0.0001)$ and the increase in CD4 cells count was 267 with DTG vs. 208 with EFV $(p<0.001)$. The main AEs observed in the DTG arm were insomnia and a mild, non-progressive 
increase in the serum creatinine without any effect on the actual glomerular filtration rate. Discontinuation due to AEs was observed in $10 \%$ of the patients in the EFV arm vs. $2 \%$ in the DTG arm and the higher discontinuation rate in the EFV arm drove the overall greater efficacy. Moreover, in patients failing cART in the DTG arm, resistance to any of the regimen components did not develop [59]. The SINGLE study supported the idea of co-formulating $\mathrm{ABC} / 3 \mathrm{TC} / \mathrm{DTG}$ as a new promising STR whose limits might be related to the backbone: restricted use to patients HLAB*5701 negative.

Dolutegravir is, since August 2013, approved in the US for the treatment of HIV-1 infection in combination with other ARV drugs, but studies exploring the potential of the $\mathrm{ABC} / 3 \mathrm{TC} /$ DTG STR are ongoing, such as the ARV treatment in ART-naïve women (ARIA Study, NCT\#01910402) [68].

Tenofovir alafenamide (TAF) is an investigational pro-drug of TDF with lower plasma concentrations and increased delivery to hepatocytes and lymphoid cells. The potential advantages of TAF vs. TDF are the reduction in AEs as TAF induces smaller changes in body mineral density (BMD) and median serum creatinine, further, higher concentration in the peripheral blood mononuclear cells (PBMCs) may overcome resistance (e.g., K65R) [69].

A $25 \mathrm{mg}$ dose of TAF has shown greater ARV activity than a standard $300 \mathrm{mg}$ dose of TDF [70]. Clinically, in Phase 2 studies in cART-naïve patients, TAF resulted in non-inferior efficacy to TDF both co-formulated with FTC/EVG/COBI.

The possibility to use small doses of TAF instead of TDF could further widen the STR options as bulky molecules such as PIs could be successfully co-formulated (e.g., FTC/TAF/ COBI/DRV and other third agents). Studies on STR including TAF such as FTC/TAF/COBI/EVG or FTC/TAF/COBI/DRV are already ongoing.
In the next few months, the patents of several relevant ARV drugs will expire and the possibility to combine bioequivalent drugs will become a reality, it has been hypothesized the possibility to obtain a fully bioequivalent STR combining $\mathrm{ABC} / 3 \mathrm{TC} / \mathrm{EFV}$.

\section{Limits of STRs in Clinical Practice}

STRs, through regimen simplification, offer major advantages in the management of HIVpositive individual, but cannot be the answer to all problems. Intrinsic to the concept of STR are some potential limitations to their use.

STRs are based on FDCs not allowing, therefore, for dose adjustment of single components unless breaking the regimen to more pills. This may be the case in patients with impaired renal function in which the need to adjust specific drug dosages exist (e.g., 3TC; FTC; TDF) [44]. The same may be true to limit the occurrence of adverse effects in populations with genetic backgrounds that reduce the metabolic pathways of specific drugs (e.g., EFV) [71].

A second limit may be the occurrence of intolerance as well as genetic predisposition to intolerance (e.g., HLAB*5701) to one of the components of the STR.

A third variable could be co-infections such as Hepatitis B that force clinicians to prefer, as far as possible, drugs able to control both HIV and hepatitis B virus (HBV) replication (FTC/ TDF and not 3TC/ABC) thus limiting the therapeutic options.

In deciding on the use of an STR, the clinician should pay attention to the resistance profile of any component of the STR itself remembering that transmitted resistance occurs mainly among NRTIs and NNRTIs [72, 73], shows a steady prevalence trend (of about $10-12 \%)[73,74]$ and is less frequent for newly 
developed compounds even if tested with high sensitivity methods [75].

A further variable to consider are drug-drug kinetic interactions that may expose the risk of a functional dual therapy if blood concentrations of one of the STR components are reduced, this might be the case of RPV and proton pump inhibitors co-administration [76] or dolutegravir and antacids co-administration [77]. Polypharmacy is a growing problem in the aging HIV population and besides exposing to the risk of drug-drug interactions, polypharmacy may exert a non-specific negative effect by reducing adherence [78] and therefore, partially vanishing the advantages of STRs.

Finally, the cost of the newest compound, often higher than older ones, may constitute a drawback to the use of STRs, forcing national regulatory agencies to put limitations on their prescriptions [79] or ceilings to their use.

\section{CONCLUSION}

One of the challenges of HIV infection management remains to encourage and enable patients to take ARV drugs correctly for a lifetime. Selection of a regimen should be individualized on the basis of virologic efficacy, toxicity, pill burden, dosing frequency, drug-drug interaction potential, resistance testing results, and comorbid conditions [43].

Simplicity of treatment is a key point and the combination of several active ARV agents in a single pill is a way to comply with the above considerations and offers potential advantages. These advantages, besides the improved allaround adherence, include a reduced risk of selective non-compliance, a reduced risk of prescription error, a reduced risk to expose the patient to general stigma by allowing an improved privacy and an increased acceptability, all of which might decrease the likelihood of treatment failure and subsequent selection of drug resistance.

Results of surveys show that patients prefer to take fewer daily pills, and look for compact easy-to-use regimens; observational and controlled studies indicate that virological and clinical outcomes are improved in individuals treated with single vs. MPRs, even among difficult-to-treat populations. In many cases, STRs also show economic benefit compared to other available regimens.

The choice of the initial ARV regimen is a cornerstone of the correct management of HIV infection as it may influence all the subsequent choices and residual options. Individualization of therapy is of utmost importance. It may be counterintuitive to claim the possibility to individualize treatment through the use of fixed-dose combinations, however, excluding infrequent cases (e.g., severe renal impairment or specific drug interactions); individualization is not based on the reduction/increment of doses, but rather on the choice of pharmacological components of the regimen. Therefore, the available STRs, based on the combination of different drug classes, allow prescribers to individualize treatment in naïve patients. As an example: TDF/FTC/COBI/EVG could be used in a wide variety of naïve subjects without limitations based on their pretreatment viral load or their immunological status. In the event of a reduced eGFR (<70 ml/min), TDF/FTC/RPV [71] appears a good alternative choice for the treatment of naïve patients, provided their baseline viral load is $\leq 100,000$ copies/mL, in the latter case TDF/ FTC/EFV could result in being the preferred STR.

A further advantage is that a switch from a given STR to another is possible, in the case of individual intolerance to a specific component 
or to further tailor cART to the individual preferences and lifestyles.

A possible limit of the STRs currently available is that they share a common backbone, thus limiting the possibility of drug sequencing in the case of selection of a viral clone resistant to one of the NRTI components. Patients forced to abandon their STR because of emergence of resistance to the backbone are generally obliged to switch to MPRs, often requiring more frequent dosing. STR combinations currently in development may change this situation but the future challenge would be to develop completely alternative STRs so as to extend the advantages of simplicity to heavily pre-treated individuals.

\section{ACKNOWLEDGMENTS}

No funding or sponsorship was received for this study or publication of this article. All named authors meet the ICMJE criteria for authorship for this manuscript, take responsibility for the integrity of the work as a whole, and have given final approval for the version to be published.

Conflict of interest. FM has served as a consultant on advisory boards for Boehringer Ingelheim, Bristol-Myers Squibb, Gilead, GlaxoSmithKline, Tibotec; he has received lecture fees from Bristol-Myers Squibb, Gilead, GlaxoSmithKline, Merck Sharp and Dome, and has received research and educational grants from Boehringer Ingelheim, Bristol-Myers Squibb, GlaxoSmithKline, Jansen-Cilag and Roche. N.A declares no conflict of interest.

Compliance with ethics. The analysis in this article is based on previously conducted studies, and does not involve any new studies of human or animal subjects performed by any of the authors.

Open Access. This article is distributed under the terms of the Creative Commons Attribution Noncommercial License which permits any noncommercial use, distribution, and reproduction in any medium, provided the original author(s) and the source are credited.

\section{REFERENCES}

1. Gallant JE, DeJesus E, Arribas JR, et al. Tenofovir DF, emtricitabine, and efavirenz vs. zidovudine, lamivudine, and efavirenz for HIV. N Engl J Med 2006; 354(3): 251-260.

2. Thompson MA, Mugavero MJ, Amico KR, et al. Guidelines for improving entry into and retention in care and antiretroviral adherence for persons with HIV: evidence-based recommendations from an International Association of Physicians in AIDS Care panel. Ann Intern Med. 2012;156(11):817-33.

3. Blasco AJ, Arribas JR, Boix V, et al. Costs and costefficacy analysis of the preferred treatments by GESIDA/National Plan for AIDS for the initial antiretroviral therapy in adult human immunodeficiency virus (HIV) infected patients in 2012. Enferm Infecc Microbiol Clin. 2012;30(6): 283-93.

4. Antinori A, Marcotullio S, Ammassari A, et al. Italian guidelines for the use of antiretroviral agents and the diagnostic-clinical management of HIV-1 infected persons. New Microbiol. 2011;34(2): 109-46.

5. Willig JH, Abroms S, Westfall AO, et al. Increased regimen durability in the era of once daily fixeddose combination antiretroviral therapy. AIDS. 2008;22(15):1951-60.

6. Panel on Antiretroviral Guidelines for Adults and Adolescents. Guidelines for the use of antiretroviral agents in HIV-1 infected adults and adolescents. Department of Health and Human Services, December 1, 2009. http://aidsinfo.nih.gov/ guidelines/html/1/adult-and-adolescent-arv-guide lines/0. Accessed Dec 2013.

7. Claxton AJ, Cramer J, Pierce C. A systematic review of the associations between dose regimens and medication compliance. Clin Ther. 2001;23(8): 1296-310. 
8. Stone VE, Jordan J, Tolson J, Miller R, Pilon T. Perspectives on adherence and simplicity for HIVinfected patients on antiretroviral therapy: selfreport of the relative importance of multiple attributes of highly active antiretroviral therapy (HAART) regimens in predicting adherence. JAIDS. 2004;36(3):808-16.

9. Chesney M. Adherence to HAART regimens. AIDS Patient Care STDS. 2003;17(4):169-77.

10. Ickovics JR, Meade CS. Adherence to antiretroviral therapy among patients with HIV: a critical link between behavioral and biomedical sciences. JAIDS. 2002;31(Suppl 3):S98-102.

11. Tam LW, Chui CK, Brumme CJ, et al. The relationship between resistance and adherence in drug-naïve individuals initiating HAART is specific to individual drug classes. JAIDS. 2008;49(3):266-71.

12. Bangsberg DR, Ragland K, Monk A, Deeks SG. A single tablet regimen is associated with higher adherence and viral suppression than multiple tablet regimens in HIV+ homeless and marginally housed people. AIDS. 2010;24(18):2835-40.

13. Maggiolo F, Airoldi M, Kleinloog HD, et al. Effect of adherence to HAART on virologic outcome and on the selection of resistance-conferring mutations in NNRTI- or PI-treated patients. HIV Clin Trials. 2007;8(5):282-92.

14. Aragão F, Vera J, Vaz Pinto I. Cost effectiveness of third agent class in treatment-naïve human immunodeficiency virus-infected patients in Portugal. PLOS one. 2012;7(9):e44774.

15. Maggiolo F, Ripamonti D, Arici C, et al. Simpler regimens may enhance adherence to antiretrovirals in HIV-infected patients. HIV Clin Trials. 2002;3:371-8.

16. DeJesus E, Ruane P, McDonald C, et al. Impact of switching virologically suppressed, HIV-1-infected patients from twice-daily fixeddose zidovudine/ lamivudine to once-daily fixed-dose tenofovir disoproxil fumarate/emtricitabine. HIV Clin Trials. 2008;9(2):103-14.

17. Maggiolo F, Ravasio L, Ripamonti D, et al. Similar adherence rates favor different virologic outcomes for patients treated with nonnucleoside analogues or protease inhibitors. Clin Infect Dis. 2005;40(1):158-63.

18. Stone VE, Jordan J, Tolson J, Miller R, Pilon T. Perspectives on adherence and simplicity for HIVinfected patients on antiretroviral therapy: selfreport of the relative importance of multiple attributes of highly active antiretroviral therapy
(HAART) regimens in predicting adherence. AIDS. 2004;36(3):808-16.

19. Parienti JJ, Bangsberg DR, Verdon R, Gardner EM. Better adherence with once-daily antiretroviral regimens: a meta-analysis. Clin Infect Dis. 2009;48(4):484-8.

20. De Jesus E, Young B, Morales-Ramirez JO, et al. Simplification of antiretroviral therapy to a singletablet regimen consisting of efavirenz, emtricitabine, and tenofovir disoproxil fumarate versus unmodified antiretroviral therapy in virologically suppressed HIV-1-infected patients. JAIDS. 2009;51(2):163-74.

21. Airoldi M, Zaccarelli M, Bisi L, et al. One-pill once-aday HAART: a simplification strategy that improves adherence and quality of life of HIV-infected subjects. Patient Prefer Adher. 2010;4:115-25.

22. Juday T, Gupta S, Grimm K, Wagner S, Kim E. Factors associated with complete adherence to HIV combination antiretroviral therapy. HIV Clin Trials. 2011;12(2):71-8.

23. Cohen CJ, Meyers JL, Davis KL. Association between daily antiretroviral pill burden and treatment adherence, hospitalisation risk, and other healthcare utilization and costs in a US medicaid population with HIV. BMJ Open. 2013;3:e003028.

24. Vera J, Aragão F, Guimarães M, Vaz Pinto I. Benefit of HAART simplification on adherence, clinical and economic outcomes. In: HIV11, Glasgow UK, November 2012. Abstract P5. http://hivarchive. com/hiv11/uploads/Adherence\%20-\%20Part\%20One. pdf. Accessed Dec 2013.

25. Taneja C, Juday T, Gertzog L, et al. Adherence and persistence with non-nucleoside reverse transcriptase inhibitor-based antiretroviral regimens. Expert Opin Pharmacother. 2012;13(15): 2111-8.

26. Cohen C, Davis K, Meyers J. Association of partial adherence to antiretroviral therapy with hospitalizations and healthcare costs in an HIV population. JIAS. 2012;15(suppl. 4):18060.

27. Antinori A, Angeletti C, Ammassari A, et al. Adherence in HIV-positive patients treated with single-tablet regimens and multi-pill regimens: findings from the COMPACT study. In: HIV11, Glasgow UK, November 2012. Abstract P145. http:// www.jiasociety.org/index.php/jias/article/view/ 18433. Accessed Dec 2013.

28. Laurence J. Treating HIV infection with one pill per day. AIDS Patient Care STDs. 2006;20(9):601-3. 
29. Glass TR, De Geest S, Weber R, et al. Correlates of self-reported non-adherence to antiretroviral therapy in HIV-infected patients. The Swiss HIV Cohort study. JAIDS. 2006;41(3):385-92.

30. Deschamps AE, De Graeve V, Van Wijngaerden E, et al. Prevalence and correlates of non adherence to antiretroviral therapy in a population of HIV patients using medication event monitoring system. AIDS Patient Care STDs. 2004;18(11):644-57.

31. Heath KV, Singer J, O'Shaughnessy MV, et al. Intentional non adherence due to adverse symptoms associate with antiretroviral therapy. JAIDS. 2002;31(2):211-7.

32. Ammassari A, Antinori A, Cozzi-Lepri A, et al. Relationship between HAART adherence and adipose tissue alterations. JAIDS. 2002;31:S140-4.

33. Horberg MA, Silverberg MJ, Hurley LB, et al. Effects of depression and selective serotonin reuptake inhibitor use on adherence to highly active antiretroviral therapy and on clinical outcomes in HIV-infected patients. JAIDS. 2008;47(3):384-90.

34. Homar F, Lozano V, Martínez-Gómez J, et al. Cost analysis of HIV treatment and drug-related adverse events when fixed-dose combinations of antiretrovirals (FDCs) were stopped, versus continuation with FDCs. Health Econ Rev. 2012;2(1):16 (Epub ahead of print).

35. Juday T, Grimm K, Willig J, Zoe-Powers A, Kim E. A retrospective study of HIV antiretroviral treatment persistence in a commercially insured population in the United States. AIDS Care. 2011;23(9):1154-62.

36. Hodder SL, Mounzer K, Dejesus E, et al. Patientreported outcomes in virologically suppressed, HIV1-infected subjects after switching to a simplified, single-tablet regimen of efavirenz, emtricitabine, and tenofovir DF. AIDS Patient Care STDs. 2010;24(2):87-96.

37. Palella F, Tebas P, Gazzard B, et al. SPIRIT study: switching boosted PI to rilpivirine in combination with truvada as a single tablet regimen. In: International AIDS conference, Washington DC, July 2012. Abstract TUAB0104. http://www.natap. org/2012/IAS/IAS_05.htm. Accessed Dec 2013.

38. Sax PE, Meyers JL, Mugavero M, Davis KL. Adherence to antiretroviral treatment and correlation with risk of hospitalization among commercially insured HIV patients in the United States. PLoS ONE. 2012;7(2):e31591.

39. Colombo GL, Di Matteo S, Maggiolo F, et al. Antiretroviral therapy in HIV-infected patients: a proposal to assess the economic value of the Single
Tablet Regimen (STR). Clinicoecon Outcomes Res. 2013;5:59-68.

40. German P, Warren D, West S, Hui J, Kearney BP. Pharmacokinetics and bioavailability of an integrase and novel pharmacoenhancercontaining single-tablet fixed-dose combination regimen for the treatment of HIV. JAIDS. 2010;55(3):323-9.

41. Cohen C, Elion R, Ruane P, et al. Randomized, phase 2 evaluation of two single-tablet regimens elvitegravir/cobicistat/emtricitabine/tenofovir disoproxil fumarate versus efavirenz/emtricitabine/ tenofovir disoproxil fumarate for the initial treatment of HIV infection. AIDS. 2011;25(6):F7-12.

42. Elion R, Gathe J, Rashbaum B, et al. The singletablet regimen elvitegravir/cobicistat/ emtricitabine/tenofovir disoproxil fumarate (EVG/ COBI/FTC/TDF; "QUAD") maintains a high rate of virologic suppression, and cobicistat (COBI) is an effective pharmacoenhancer through 48 weeks. In: 50th ICAAC 2010, Boston, USA. Abstract PH-938-B. http://www.hivandhepatitis.com/2010_conference/ icaac/posters/Quad.pdf. Accessed Dec 2013.

43. Panel on Antiretroviral Guidelines for Adults and Adolescents. Guidelines for the use of antiretroviral agents in HIV-1 infected adults and adolescents. Department of Health and Human Services; December 2013. http://aidsinfo.nih.gov/guidelines/ html/1/adult-and-adolescent-arv-guidelines/0. Accessed Jan 2014.

44. European AIDS Clinical Society. Guidelines for the Clinical management and Treatment of HIV Infected Adults in Europe. Version 7.0, Oct 2013. http://www.eacsociety.org/Guidelines.aspx. Accessed Jan 2014.

45. Antinori A, Marcotullio S, Ammassari A, et al. Italian guidelines for the use of antiretroviral agents and the diagnostic-clinical management of HIV-1 infected persons (November 2013). http:// www.salute.gov.it/imgs/C_17_pubblicazioni_1793_ allegato.pdf. Accessed Jan 2014.

46. Moyle G, Orkin C, Fisher M, et al. Switching to a single-tablet regimen (STR) of Atripla ${ }^{\circledR}$ (ATR) from a 2 or 3-pill combination of the individual components (efavirenz [EFV], emtricitabine[FTC] and tenofovir df [TDF]) maintains virological suppression: primary endpoint results of a 48-week, open-label study. HIV Med. 2011;12:79.

47. Deeks ED, Perry CM. Efavirenz/emtricitabine/ tenofovir disoproxil fumarate single-tablet regimen $\left(\right.$ Atripla ${ }^{\circledR}$ ): a review of its use in the management of HIV infection. Drugs. 2010;70(17):2315-38. 
48. De Jesus E, Rockstroh JK, Henry K, et al. Coformulated elvitegravir, cobicistat, emtricitabine, and tenofovir disoproxil fumarate versus ritonavirboosted atazanavir plus co-formulated emtricitabine and tenofovir disoproxil fumarate for initial treatment of HIV-1 infection: a randomized, double blind, phase 3, noninferiority trial. Lancet. 2012;379:2429-38.

49. Rockstroh JK, De Jesus E, Henry K, et al. A randomized, double-blind comparison of coformulated elvitegravir/cobicistat/emtricitabine/ tenofovir versus ritonavir-boosted atazanavir plus co-formulated emtricitabine and tenofovir DF for initial treatment of HIV-1 infection: analysis of week 96 results. JAIDS. 2013;62(5):483-6.

50. Charpentier C, Lambert-Niclot S, Visseaux B, et al. Evolution of the K65R, K103N and M184V/I reverse transcriptase mutations in HIV-1-infected patients experiencing virological failure between 2005 and 2010. JAC. $201 ; 68(10): 2197-8$.

51. Ortega-Gonzales E, Garcia Deltoro M, LopezAldeguerJ, et al. Trend and prevalence of HIV-1 resistance mutations in the Valencian Autonomous Region (2004-2011) and its relation with the antiretroviral usage pattern: RUVEN study (SEICVVIH-2012-01). In: 14th EACS, Brussels Belgium, October 2013. Abstract PE9/28. http://www. abstracttosubmit.com/eacs2013/eposter/. Accessed Feb 2014.

52. Cohen CJ, Molina JM, Cahn P, et al. Efficacy and safety of rilpivirine versus efavirenz at 48 weeks in treatment-naïve HIV-infected patients: pooled results from the phase 3 double-blind randomized ECHO and THRIVE trials. JAIDS. 2012;60(1):33-42.

53. Clumeck N, Molina JM, Henry $\mathrm{K}$, et al. A randomized, double-blind comparison of single tablet regimen elvitegravir/cobicistat/ emtricitabine/tenofovir DF versus ritonavirboosted atazanavir plus emtricitabine/tenofovir DF for initial treatment of HIV-1 infection: analysis of week 144 results. J Acquir Immune Defic Syndr. 2013;16 [Epub ahead of print].

54. Eron J, Rockstroh J, Pozniak A, et al. Dolutegravir treatment response by baseline viral load and NRTI backbone in treatment-naïve HIV-infected individuals. In: HIV11, Glasgow UK, November 2012. Abstract P204. http://www.natap.org/2012/ interHIV/InterHIV_03.htm. Accessed Dec 2013.

55. Raffi F, Jaeger H, Quiros-Roldan E, et al. Once daily dolutegravir versus twice daily raltegravir in antiretroviral-naïve adults with HIV-1 infection (SPRING-2 study):96 week results from a randomized, double-blind, non-inferiority trial. Lancet Infect Dis. 2013;13(11):927-35.
56. Feinberg J, Clotet B, Khuong MA, et al. Once-daily dolutegravir is superior to darunavir/ritonavir in antiretroviral naive adults: 48 week results from Flamingo. In: 53rd ICAAC, Denver USA, September 2013. Abstract H1464a. http://www.natap.org/ 2013/ICAAC/ICAAC_24.htm. Accessed Dec 2013.

57. Cohen C, Wohl D, Arribas J et al. STaR study: single tablet regimen emtricitabine/rilpivirine/tenofovir is non-inferior to efavirenz/emtricitabine/tenofovir DF in ART-naïve adults. In: HIV11, Glasgow UK, November 2012. Abstract O425. http://www. jiasociety.org/index.php/jias/article/view/18221. Accessed Dec 2013.

58. Cohen CJ, Molina JM, Cassetti I, et al. Week 96 efficacy and safety of rilpivirine in treatment-naive, HIV-1 patients in two Phase III randomized trials. AIDS. 2013;27(6):939-50.

59. Nelson M, Winston A, Waters L, et al. Multicentre open-label study of switching from atripla to eviplera for possible efavirenz associated CNS toxicity. In: 53rd ICAAC, Denver USA, September 2013. Abstract H-672-b. http://www.natap.org/ 2013/ICAAC/ICAAC_47.htm. Accessed Dec 2013.

60. Mills AM, Cohen C, Dejesus E, et al. Efficacy and safety 48 weeks after switching from efavirenz to rilpivirine using emtricitabine/tenofovir disoproxil fumarate-based single-tablet regimens. HIV Clin Trials. 2013;14(5):2216-355.

61. Panel on Antiretroviral Guidelines for Adults and Adolescents. Recommendation on integrase inhibitor use in antiretroviral treatment-naïve HIV-infected individuals from HHS Panel on Antiretroviral Guidelines for Adults and Adolescents Department of Health and Human Services; October 30, 2013. http://aidsinfo.nih. gov/contentfiles/upload/adultARV_INSTIRecommen dations.pdf. Accessed Jan 2014.

62. Molina JM, Lamarca A, Andrade-Villanueva J, et al. International, randomized, double blinded, 96-week, non-inferiority study of EVG QD versus RAL BID in ARV-experienced patients. Lancet Infect Dis. 2012;12(1):27-35.

63. Cahn P, Pozniak AL, Mingrone H, et al. Dolutegravir versus raltegravir in antiretroviral-experienced, integrase-inhibitor-naive adults with HIV: week 48 results from the randomised, double-blind, noninferiority SAILING study. Lancet. 2013;382:700-8.

64. Sax PE, De Jesus E, Mills A, et al. Co-formulated elvitegravir, cobicistat, emtricitabine, and tenofovir versus co-formulated efavirenz, emtricitabine, and tenofovir for initial treatment of HIV-1 infection: a randomized, double-blind, phase 3 trial, analysis of results after 48 weeks. Lancet. 2012;379:2439-48. 
65. Zolopa A, Sax PE, DeJesus E, et al. A randomized, double-blind comparison of co-formulated elvitegravir/cobicistat/emtricitabine/tenofovir DF versus efavirenz/emtricitabine/tenofovir DF for initial treatment of HIV-1 infection: analysis of week 96 results. JAIDS. 2013;63(1):96-100.

66. Walmsley SL, Antela A, Clumeck $\mathrm{N}$, et al. Dolutegravir plus abacavir/lamivudine for the treatment of HIV-1 infection. $\mathrm{N}$ Engl J Med. 2013;369(19):1807-18.

67. Wohl DA, Cohen C, Gallant JE, et al. A randomized, double-blind comparison of single tablet regimen elvitegravir/cobicistat/emtricitabine/tenofovir DF versus single tablet regimen efavirenz/ emtricitabine/tenofovir DF for initial treatment of HIV-1 infection: analysis of week 144 results. JAIDS. 2013;19 [Epub ahead of print].

68. A study to determine safety and efficacy of dolutegravir/abacavir/lamivudine (DTG/ABC/3TC) in human immunodeficiency virus (HIV)-1 infected antiretroviral therapy (ART) Naïve women (ARIA). http://clinicaltrials.gov/show/NCT01910402? order $=0$. Accessed Jan 2014.

69. Zolopa A, Ortiz R, Sax P, et al. Comparative study of tenofovir alafenamide vs tenofovir disoproxil fumarate, each with elvitegravir, cobicistat and emtricitabine for HIV treatment. In: 20th CROI, Atlanta USA, March 2013. Abstract 99LB. http:// www.natap.org/2013/CROI/croi_23.htm. Accessed Dec 2013.

70. Ruane PJ, DeJesus E, Berger D, et al. Antiviral activity, safety, and pharmacokinetics/ pharmacodynamics of tenofovir alafenamide as 10-day monotherapy in HIV-1-positive adults. J Acquir Immune Defic Syndr. 2013;63(4):449-55.

71. King J, Aberg JA. Clinical impact of patient population differences and genomic variation in efavirenz therapy. AIDS. 2008;22:1709-17.

72. Schmidt D, Kollan C, Fatkenheuer $G$ et al. Proportion of transmitted and acquired HIV drug resistance in a long term observational cohort in Germany. In: 14th European AIDS conference.
Brussels, Belgium, October 2013. PE9/11. http:// www.eacs-conference2013.com/fileadmin/templates/ eacs/template_FILES/FINAL_EACS13_Final_Program_ web.pdf. Accessed Dec 2013.

73. Batmeyer B, Kuecherer C, Houareau C, et al. Prevalence of transmitted drug resistance and impact of transmitted resistance on treatment success in the German HIV-1 seroconverter cohort. PLoS ONE. 2010;5(10):e12718.

74. Meixenberger K, Scheufele R, Jansen K, et al. In vivo prevalence of transmitted drug-resistant HIV in patients with a known date of HIV-1 seroconversion. In: 14th European AIDS conference. Brussels, Belgium, October 2013. PE9/ 24. http://www.eacs-conference2013.com/fileadmin/ templates/eacs/template_FILES/FINAL_EACS13_ Final_Program_web.pdf. Accessed Dec 2013.

75. Chueca N, Camacho-Luque R, Martinez NM, et al. Prevalence of low abundant rilpivirine resistance associated mutations in naive patients from the south of Spain. In: 14th European AIDS Conference. Brussels, Belgium, October 2013. PE9/16. http:// www.eacs-conference2013.com/fileadmin/templates/ eacs/template_FILES/FINAL_EACS13_Final_Program_ web.pdf. Accessed Dec 2013.

76. Crauwels $H$, van Heeswijk RP, Stevens M, et al. Clinical perspective on drug-drug interactions with the non-nucleoside reverse transcriptase inhibitor rilpivirine. AIDS Rev. 2013;15(2):87-101.

77. Sha BM, Schafer JJ, DeSimone JA. Dolutegravir: a new integrase strand transfer inhibitor for the treatment of HIV. Pharmacotherapy. 2013;18 [Epub ahead of print].

78. Edelman EJ, Gordon KS, Glover J, McNicholl IR, Fiellin DA, Justice AC. The next therapeutic challenge in HIV: polypharmacy. Drugs Aging. 2013;30:613-28.

79. NHS England Clinical Reference Group. Clinical Commissioning policy statement: stribild for the treatment of HIV-1 infection in adults. http://www. england.nhs.uk/wp-content/uploads/2013/09/b06psa1.pdf. Accessed Jan 2014. 